\title{
The cost of accuracy in crop area estimation
}

\author{
Hugo De Groote ${ }^{\mathrm{a}, *}$, Oumar Traoré ${ }^{\mathrm{b}, 1}$ \\ a International Maize and Wheat Improvement Center (CIMMYT), P.O. Box 25171, Nairobi, Kenya \\ ${ }^{\mathrm{b}}$ International Crops Research Institute for the Semi-Arid Tropics, BP 320 Bamako Mali
}

Received 28 May 2003; revised 4 May 2004; accepted for publication 1 June 2004

\begin{abstract}
Accuracy and cost of direct crop area measurement are compared with those of farmers' estimates after visual inspection, in this methodological study conducted in southern Mali. The observational error, the difference between the area measured and the area estimated, was first studied at the plot level. Average observational error or bias was $-11 \%$ of the average area measured, indicating an average underestimation of plots by $11 \%$. This observational error is strongly related to plot size, with smaller plots being overestimated and larger plots underestimated, in an approximately negative linear relationship. The observational error is also smaller for cotton fields than for cereals. The analysis was repeated at the farm level, where the bias in estimating the total area per farm was $-8 \%$. At this level, total error or accuracy was calculated by the relative total error (RTE), the square root of the mean square error, divided by the mean. The farmers' estimate was found to be less accurate ( RTE $=9.4 \%$ of the mean) than physical measurement $(\mathrm{RTE}=6.6 \%)$, but at a cost of only $\$ 370$ as compared with $\$ 2328$ (for a sample of 96 farms in 11 villages). The coefficient of variation (CV) of most surface variables was found to lie between $60 \%$ and $100 \%$, and their relative bias (average observational error divided by the mean area) between $2 \%$ and $10 \%$. For crop area per farm, the physical measurement of plots resulted in a gain of accuracy of $2-4 \%$, as compared with the farmers' estimate after visual inspection. A general model was developed in which these calculated parameters are used to predict the accuracy in future surveys and to compare the accuracy with the survey's cost. It is shown how the survey design can be optimized based on acceptable error, sample size and cost for each measurement technique.
\end{abstract}

\footnotetext{
${ }^{*}$ Corresponding author. Tel.: 254-20-524600; fax: 254-20-524601.

E-mail addresses: h.degroote@cgiar.org (H. De Groote), o.traore@icrisatml.org (O. Traoré).

${ }^{1}$ Previously Institut d'Economie Rural, Sikasso, Mali.
} 
Simulations demonstrate that the total error for biased estimators, even for variables with small CVs, hardly decreases above sample sizes of 100-150 farmers.

(C) 2004 Elsevier Ltd. All rights reserved.

Keywords: Area estimation; Crop area; Error; Survey accuracy; Cost

\section{Introduction}

Good crop area estimates are of major importance to agricultural statistics and to natural resource management. Precise agricultural statistics, in particular surface area and production data, are essential to the understanding of rural communities, and necessary for developing agricultural policy as well as agricultural research strategy (Kelly et al., 1995; FAO, 1982). The management of natural resources, of which land is one of the most important, is receiving increasing attention of development agencies and decision makers. To develop and to evaluate natural resource management, the monitoring of land use and therefore the measuring of surface areas are essential.

Several manuals treat the estimation of agricultural areas, among others those by the Food and Agricultural Organization (FAO, 1982) and the World Bank (Idaikkadar, 1979; Casley and Lury, 1981; Poate and Casley, 1985). One of the simplest and cheapest estimation methods described here is the farmer interview, in which a representative sample of farmers is asked to estimate the area of their fields. This method, however, faces several problems in developing countries. Farmers can be very suspicious of enumerators, especially when they perceive that the solicited information could be used for tax purposes. Moreover, in many areas where land markets are just emerging, there has been no need for area units until recently, and therefore precise local measurement units have not yet been developed. Finally, farmers generally have little access to formal education or agricultural education, where they can acquire measurement techniques and quantitative skills. Given all these difficulties, the accuracy of statistics obtained through farmers' estimates of land area is often considered to be insufficient (FAO, 1982). The FAO's experience with farmers' area estimations was not very satisfying and they recommend physical measurement (FAO, 1982), although methodological studies on the topic are scarce. On the other hand, evidence is growing that farmers' production estimates are quite accurate, and their errors similar to those of crop cutting methods (Poate, 1988; Murphy et al., 1991; Ajayi and Waibel, 2000). The World Bank ordered a methodological study in five African countries (Verma et al., 1988), which confirms the accuracy of farmers' production estimates, but shows that the quality of their area estimates depends on their familiarity with the concept and therefore varies greatly between countries. This quality is also greatly affected by the size of the unit used (e.g. $\mathrm{m}^{2}$ versus ha) and the resulting rounding error. Ajayi and Waibel (2000) note that the accuracy of farmers' estimates decreases with plot size, an observation also made by Smith et al. (1988) (cited by Ajayi and Waibel, 2000). 
The alternative to farmers' estimates is to measure areas directly, either by geographic methods such as remote sensing or sampling based on maps, or by agricultural surveys based on a representative sample of farms. In this last method, the fields of each farm of the sample are measured and the results extrapolated for the regions under consideration. In Mali, the agricultural statistics are gathered this way by the Direction Nationale de la Statistique et Informatique (DNSI, 1996) and by the cotton marketing board, the Compagnie Malienne du Developpement des Textiles (Giraudy, 1994). Similar surveys take place in most West-African countries.

For several years now, the Farming System Research team (Equipe Systèmes de Production et Gestion des Ressources Naturelles, ESPGRN) of the Malian Institut d'Economie Rurale (IER) has been conducting action research in eleven villages of the Sikasso region. A field worker is based permanently in each village to monitor field trials and to conduct farm surveys. These surveys are conducted on a regular basis to analyze major constraints and set research priorities, as well as to monitor trends in the farming system. These surveys are quite labour intensive and, given the work load of the field workers, extra enumerators are hired to measure crop areas. External funding for the Farming Systems Research (FSR) team is likely to decrease in the near future, so the team is reviewing the relevance of its wide variety of activities, in particular the farm surveys that take a considerable amount of resources. The present study compares the currently used physical area measurement method with a much cheaper, but probably less accurate method, in which the farmer was assisted by the enumerator, and both visited the fields together to estimate the area of each plot. Although these visits increased the cost of the survey, they did ensure that all fields were included. Moreover, they allowed the enumerator to point out inconsistencies in the farmers' estimates and thus improve accuracy.

The objectives of this study are therefore: (i) to compare the accuracy of area measurement with the accuracy of farmers' estimates, assisted by enumerators, (ii) to compare the costs of the two methods, and (iii) to analyze the relationship between accuracy and cost and make appropriate recommendations.

\section{The model}

\subsection{A general error model for linear estimators}

A natural and intuitive measure of estimation error is the expected value of the difference between the estimate and the estimated parameter, squared so that positive and negative errors do not cancel each other out. This measure is called the mean squared error (MSE); it can also be seen as a loss function, and is commonly used as such (Cochran, 1977). Mathematically, the MSE of an estimator $T$ for a population statistic $\theta$ can be decomposed as

$$
\operatorname{MSE}(T)=E(T-\theta)^{2}=V(T)+[\theta-E(T)]^{2} .
$$


The MSE can thus be regarded as the combination of the variance $V(T)$ and the bias $B=\theta-E(T)$, which makes it a useful criterion to compare biased estimators (Cochran, 1977). The MSE can therefore also be interpreted as the total survey error (Groves, 1989) and is a measure of the accuracy of the estimator. The variance around the sample mean $V(T)$ is usually referred to as precision, and does not include the bias (but it does include other non-sampling errors). Although precision is often used as a criterion for the quality of survey designs, MSE is a superior criterion because it evaluates both sampling and non-sampling errors, including bias. Non-sampling errors include errors such as the measurement error of the measuring instruments used, the difference between real and observed values, and the errors made by estimation. These are all very common errors in field surveys.

In order to develop a practical model, each variable in the survey data can be denoted by a vector $\boldsymbol{y}$, containing one observation for each sample element. This vector can be separated into a vector of true values of the measured characteristic $\boldsymbol{x}$ and a vector of observational errors $\boldsymbol{e}$

$$
\boldsymbol{y}=\boldsymbol{x}+\boldsymbol{e} .
$$

Let $\boldsymbol{x}$ be distributed around a vector of means $\boldsymbol{\mu}$ and with a variance-covariance matrix $\Sigma$, or $\boldsymbol{x} \sim(\boldsymbol{\mu}, \Sigma)$, and let $\boldsymbol{e} \sim(\boldsymbol{\beta}, \Omega)$. The matrix of covariances between the elements of $\boldsymbol{x}$ and $\boldsymbol{e}$ can be denoted as the matrix $\Phi_{x e}$, and the matrix of covariances between $\boldsymbol{e}$ and $\boldsymbol{x}$ as $\Phi_{e x}$. Assuming covariance $\left(x_{i}, e_{\mathrm{j}}\right)=\operatorname{covariance}\left(x_{i}, e_{\mathrm{j}}\right), \Phi_{e x}=\Phi_{x e}$. This is a generalization of the basic additive error model (Cochran, 1977, p. 377; Groves, 1989) in vector notation. Most estimators in survey statistics are a linear function of a sample of observations, in vector notation

$$
T=\boldsymbol{h}^{\prime} \boldsymbol{y},
$$

where $\boldsymbol{h}$ is a vector of weights. It follows from the general formula (1) that

$$
\operatorname{MSE}(T)=\boldsymbol{h}^{\prime}\left(\Sigma+2 \Phi_{x e}\right) \boldsymbol{h}+\left(\boldsymbol{h}^{\prime} \boldsymbol{\mu}+\boldsymbol{h}^{\prime} \boldsymbol{\beta}-\theta\right)^{2} .
$$

This model can encompass most sampling and non-sampling errors, and unlike other models it makes the study of their combined effects possible (De Groote, 1996).

\subsection{A simple error model}

For the purpose of this methodological study, we can use a simpler model. Assume a sample of $n$ observations, grouped in a vector $\boldsymbol{y}$. Each element $y_{i}$ is the sum of the true value $x_{i}$ and the observation error $e_{i}$, and the elements of each vector are identically and independently distributed, but there is a correlation between true value $x_{i}$ and error $e_{i}$. Mathematically, the true values $x_{i}$ have a mean $\mu$ (so all elements of the vector $\boldsymbol{\mu}$ have the same value $\mu$ ), and variance of each $x_{i}$ is $\sigma_{x}^{2}$ (so the diagonal elements of the variance covariance matrix $\Sigma$ have the value $\sigma_{x}^{2}$, while the other elements are equal to 0 ). Similarly, each error element $e_{i}$ has a mean $B$ or bias (so all elements of the vector $\beta$ have the same value $B$, conventionally the symbol for bias) and a variance of $\sigma_{e}^{2}$ (diagonal elements of $\Omega$ ). The covariance between true value and error of each element is $\sigma_{x e}$ (diagonal elements of the matrix $\Phi_{x e}$ ) but it 
is assumed there is no correlation between true values and errors of different elements (so the other elements of the matrices are zero). Formally, each pair is now equally and independently distributed, following the same bivariate distribution

$$
\left(x_{i}, e_{i}\right) \sim\left(\mu, B, \sigma_{x}^{2}, \sigma_{e}^{2}, \sigma_{x e}\right) .
$$

A characteristic of the population is estimated with a linear estimator $T$, in our case the sample mean

$$
T=\bar{y}=\boldsymbol{h}^{\prime} \boldsymbol{y}, \quad \text { where } \boldsymbol{y}=\left(\begin{array}{c}
y_{1} \\
\cdot \\
y_{i} \\
\cdot \\
y_{n}
\end{array}\right) \text { and } \quad \boldsymbol{h}=\left(\begin{array}{c}
1 / n \\
\cdot \\
1 / n \\
\cdot \\
1 / n
\end{array}\right) .
$$

The total error or accuracy of the sample mean as an estimator of the population mean can now be derived from the general formula (4), by entering the specifications into the vectors and matrices, resulting in the simple formula

$$
\operatorname{MSE}(\bar{y})=\sqrt{\frac{\left(\sigma_{x}^{2}+\sigma_{e}^{2}+2 \sigma_{x e}\right)}{n}+B^{2}}
$$

We would like to introduce a new and convenient concept, the relative total error or RTE, analogous to the coefficient of variation (CV). To calculate the CV, one divides the square root of the variance by the mean to obtain a relative measure of variance, free of units. Similarly, we can define the relative total error as the square root of the MSE (which represents total error) divided by the mean of the population

$$
\operatorname{RTE}(\bar{y})=\sqrt{\frac{\sigma_{x}^{2}+\sigma_{e}^{2}+2 \sigma_{x e}}{n \mu^{2}}+\frac{B^{2}}{\mu^{2}}} .
$$

Expressing the total error as a percentage of the mean makes its interpretation much easier, as well as comparison of methods used in different circumstances. In similar fashion, the bias $B$ can be divided by the mean to obtain the relative bias BR. The standard deviations, the covariance and the bias can also be expressed as percentages of the population mean, and represented by, respectively, $\mathrm{SR}_{x}, \mathrm{SR}_{e}$, and $\mathrm{SR}_{x e}$. The formula for RTE becomes

$$
\mathrm{RTE}=\sqrt{\frac{\mathrm{SR}_{x}^{2}+\mathrm{SR}_{e}^{2}+2 \mathrm{SR}_{x e}}{n}+\mathrm{BR}^{2} .}
$$

The standard deviation divided by the mean, $\mathrm{SR}_{e}$, is normally called the coefficient of variation or $\mathrm{CV}$, but was renamed here for consistency in notation. As will be shown next, these four elements can be collected through a methodological study, resulting in the calculation of the total error. Because all elements are unit free, their values are particularly attractive to be used as approximations for future surveys or surveys in other regions. 
The empirical section of this paper only deals with the RTE of the sample mean, generally the most important statistic in sample surveys. However, many surveys also seek to quantify relationships, most often through the calculation of regression coefficients. The general formula of Eq. (4) holds for all linear estimators, including regression coefficients for non-stochastic independent variables. The optimization of panel data surveys to estimate an evolution over time is another straight-forward application (De Groote, 1992).

\section{Study area, data and methods}

The FSR team of Sikasso is active in the administrative region of Sikasso, which has an average yearly rainfall of $800-1200 \mathrm{~mm}$. The agricultural system is based on cereal production, mostly for home consumption, and cotton as a cash crop. Population is estimated at 21.4 people $/ \mathrm{km}^{2}$, with an annual population growth of $2.3 \%$ (DNSI, 1996). Farm households consist on average of 14 members, and cultivate $9.5 \mathrm{ha}$, of which 6.6 ha is in cereals and 1.7 ha is in cotton (Kébé et al., 1997). Surplus revenue is generally invested in livestock, and, on average, 9 cattle and 8 small ruminants buffer the household against the highly variable crop production.

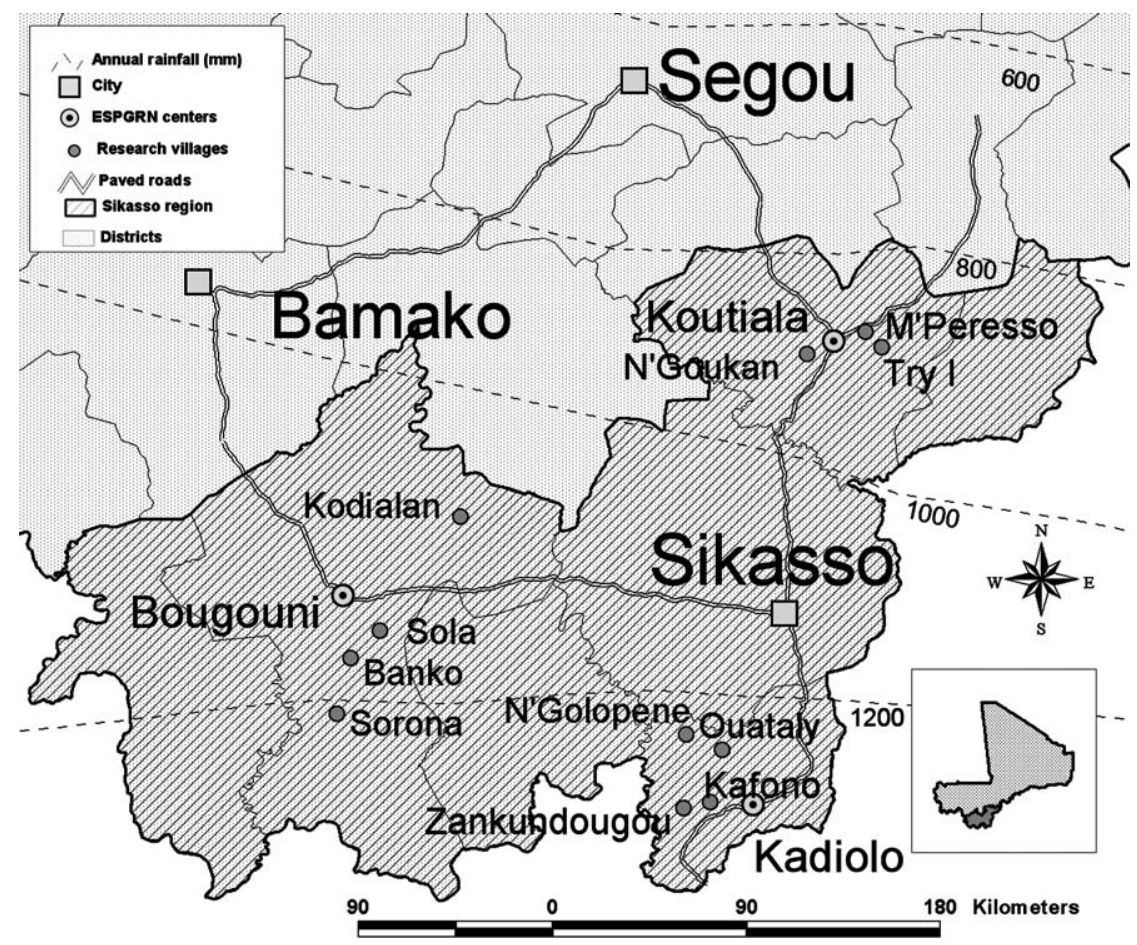

Fig. 1. Map of the Farming Systems Research zone with research villages. 
At the time of the survey, the FSR team was working in 11 research villages (see map in Fig. 1), with a field worker or enumerator in each of these villages. Every year these field workers collect data on 96 farms, between 8 and 12 per village. During 1994, a new area estimation technique was tested. For this test, each enumerator visited all the fields of the sample farms in his or her village, together with the farmer, to obtain the farmers' estimate of their size, in ha. After visual inspection by the enumerator, the initial estimate of the farmer was discussed if needed, and a consensus estimate retained.

During the same season, another team of two enumerators visited all the fields of the 96 sample farms and measured each plot, following an FAO procedure (FAO, 1982, p. 58). An outline of each plot was drawn on a special surveyor's board "Chaix" (Ministère de la Coopération, 1980, p. 179). The length of each polygon side was measured by an instrument called "topofil" (Ministère de la Coopération, 1980, p. 173), which unwinds a disposable line from a spool between two points, while measuring and indicating its length on a precision counter. The orientation of the polygon side was measured in geographic degrees with a compass. From the length and the orientation of all sides, the polygon's surface area was calculated with a programmable pocket calculator according to standard calculation methods (FAO, 1982).

For each plot, the area measurement and the visual estimation was entered in a computer data base, together with an identification code and other plot data such as crop rotation and input use. In total, the areas of 1168 plots were measured, and those of 1098 plots estimated after visual inspection. After an initial cleaning of the data, 18 more observations were discarded: the difference between observed and measured was larger than 6 times the measured value, and it was assumed that this discrepancy was due to a writing or typing error. This left a database of 1080 observations.

The observational error for each plot was calculated as the difference between the area resulting from the physical measurement (by the topofil method, of which the measurement error was assumed to be negligible), and the area resulting from estimation after visual inspection. This observational error is distinct from the commonly used term "recall error", which indicates errors due to an inaccurate memory of an event or a quantity. Since farmers and enumerators actually visited the field, the term recall would not be appropriate.

To analyze the data, first the errors in estimating plot size were calculated and the effect of the major factors analyzed. Because most data of interest are situated at the farm level, however, the plot data were aggregated by farm and by current season crop. Subsequently, the observational errors were recalculated at this aggregated level, and the factors influencing the observational error were analyzed again at this level.

The observational errors were then combined with the sampling error in the calculation of the total survey error, as expressed by the RTE. The RTE was analyzed for both the physical measurement method and for the visual estimation method, at the plot level as well as at the farm level, and for all major crops. Finally, the costs of both methods of quantifying agricultural areas were calculated and compared with their accuracy. The results were then used to construct a general but simple model, that calculates the accuracy of measurement error based on a few essential, unit-free parameters. 


\section{Average observational errors or bias}

\subsection{Bias in estimating plot size}

The physical measurement of the plots, through the topofil method, resulted in an average surface area of 0.816 ha. Plot size varies between 0.01 and 8.78 ha, with a coefficient of variation of $124 \%$ (first line in Table 1). The farmers' estimates of the same plots, after visual inspection, resulted in an average plot size of 0.723 ha. The difference between the physical measurement and the farmers' estimate produces a value for the observational error $\left(e_{i}\right.$ from Eq. (5)). The mean of those observational errors is an estimate of the bias, and equals -0.093 ha (second line in Table 1). Dividing this bias by the average measured plot size results in the relative bias (BR) of $-11.4 \%$, indicating that on average plot sizes are underestimated by $11 \%$. Although the observational error is relatively small on average, it is highly variable, ranging from an underestimation of 4.25 ha to an overestimate of 2.17 ha.

The observational error is clearly related to plot size, as well as to the crop grown on this plot. Fig. 2 shows that the bias is positive for plots smaller than 1 ha, meaning that farmers show a tendency to overestimate small plots. Larger plots, on the other hand, are underestimated, and the underestimation increases with plot size. The observational error is clearly smaller in cotton fields. These fields have often been measured in the past by an enumerator of the cotton company, and its area is important to calculate fertilizer and pesticide applications.

As Fig. 2 demonstrates, this relationship between estimation error and surface area, both expressed in ha, is approximately linear. Estimated by regression analysis, using individual plots as observations but allowing for a different slope for cotton plots, the relationship becomes (standard deviation of the coefficients in brackets)

$$
\begin{aligned}
\text { observational error }= & 0.14(0.016)-0.31(0.014) \text { area }+0.14(0.022) \text { cotton } \\
& \times \text { area }\left(R^{2}=0.33, \text { std. error }=0.42, N=1080\right) .
\end{aligned}
$$

This equation indicates that with every ha of plot size, the surface area is underestimated by a third of a hectare $(0.31)$ for crops other than cotton. For plots under cotton, the underestimation increases by 0.17 ha per hectare $(-0.31+0.14)$. Coefficients for other crops were not significantly different from zero.

The average observational error or bias was also calculated by crop. The results are presented in Table 2 and show again that the absolute value of the relative bias for cotton fields, $-7.8 \%$, is smaller than for cereals, $-14 \%$. The bias in legumes is

Table 1

Observational errors, plot level results

\begin{tabular}{lrrrrl}
\hline Variable & Mean & Min & Max & SD & \multicolumn{1}{l}{$N$} \\
\hline Measured plot size (ha) & 0.816 & 0.01 & 8.78 & 1.06 & 1080 \\
Estimated plot size (ha) & 0.723 & 0.01 & 8.00 & 0.88 & 1080 \\
Observational error (ha) & -0.093 & -4.25 & 2.17 & 0.50 & 1080 \\
Relative observational error (\% of the average plot size) & -11.4 & & & & \\
\hline
\end{tabular}




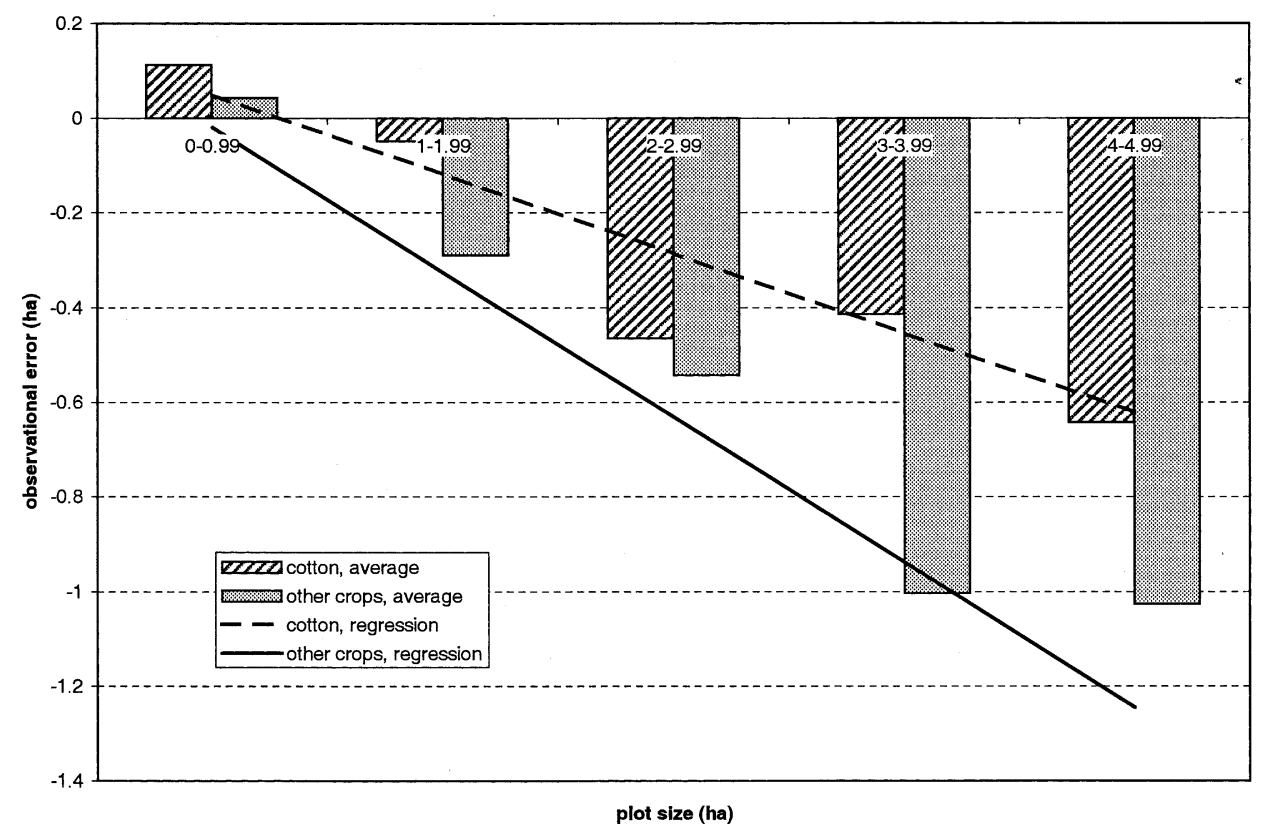

Fig. 2. Observational error in estimating field areas, by plot size and crop.

Table 2

Observational error in plot size, by crop

\begin{tabular}{|c|c|c|c|c|c|c|}
\hline \multirow[t]{2}{*}{ Crop } & \multicolumn{2}{|c|}{$\begin{array}{l}\text { Area measured } \\
\text { (ha) }\end{array}$} & \multicolumn{2}{|c|}{$\begin{array}{l}\text { Observational error } \\
\text { (ha) }\end{array}$} & \multirow[t]{2}{*}{ Relative bias BR (bias/mean) } & \multirow[t]{2}{*}{$N$} \\
\hline & Mean & SD & Mean (bias) & SD & & \\
\hline Cereals & 0.86 & 1.07 & -0.121 & 0.57 & -0.141 & 735 \\
\hline Cotton & 1.67 & 1.38 & -0.130 & 0.49 & -0.078 & 110 \\
\hline Legumes & 0.34 & 0.35 & -0.003 & 0.18 & -0.008 & 169 \\
\hline Other crops & 0.15 & 0.14 & 0.039 & 0.13 & 0.254 & 66 \\
\hline Total & 0.82 & 1.06 & -0.093 & 0.50 & -0.114 & 1080 \\
\hline
\end{tabular}

particularly small, not even $-1 \%$. All other crops, typically grown on small plots, show an overestimation of $25 \%$ on average. The difference between crops can largely be explained by the difference in average plot size, except for cotton. The absolute value of the bias for cotton, with an average plot size of $1.24 \mathrm{ha}$, is smaller than that for cereals, with an average plot size of 0.48 ha, but this is understandable since farmers usually know the size of their cotton plot as measured by the extension agents.

Because all plots in one village were estimated with the help of the same enumerator, the error differences between villages give an indication of the human factor or enumerator error. Although differences between villages were observed, multiple 
regression analysis showed that they were not significant once the differences in plot size were taken into account. The interpretation is that certain villages just have a better balance between small plots (and their overestimation) and bigger plots (with underestimates).

\subsection{Bias in the estimation of total area}

So far, only plot size was treated. In practice, however, we are usually interested in variables such as total farm area under cultivation, or cotton area per farm. These farm-level data are the backbone of agricultural statistics: they are used to extrapolate and calculate regional or national cultivated areas and, in combination with yield estimates, production.

For each farm in the sample, the total area measured was calculated by summing up the plot sizes measured, and similarly the total area estimated. The results, presented in Table 3, show that the aggregation of overestimated small plots and underestimated large plots decreases total error at the farm level. Overall, total area per farm is underestimated by $8 \%$. The relative bias is similar for area per farm under cotton $(-7.8 \%)$ and for cereals $(-9.5 \%)$. The bias is again small for legumes and very large for all other crops combined.

\section{Cost versus accuracy}

\subsection{Calculating the accuracy through the total survey error}

To calculate total survey error, we have to combine the observational errors $\left(e_{i}\right.$ from formula (5)) treated in the previous section, with sampling errors. This can conveniently be achieved by using the relative total error (RTE), calculated with Eq. (9). The components of this formula are the relative standard deviation of the area variable $\mathrm{SR}_{x}$ (standard deviation/mean or coefficient of variation), the relative standard deviation of the observational error $\mathrm{SR}_{e}$, their relative covariance $\mathrm{SR}_{x e}$ (covariance/ mean $^{2}$ ), and relative bias BR.

Table 3

Observational error in estimating farm area, total and by crop

\begin{tabular}{|c|c|c|c|c|c|c|}
\hline \multirow[t]{2}{*}{ Crop } & \multicolumn{2}{|c|}{$\begin{array}{l}\text { Area measured } \\
\text { (ha/farm) }\end{array}$} & \multicolumn{2}{|c|}{$\begin{array}{l}\text { Observational } \\
\text { error (ha/farm) }\end{array}$} & \multirow[t]{2}{*}{ Relative bias BR (bias/mean) } & \multirow[t]{2}{*}{$N$} \\
\hline & Mean $\mu$ & $\mathrm{SD} \sigma_{x}$ & Mean $B$ & $\mathrm{SD} \sigma_{e}$ & & \\
\hline Cereals & 6.7 & 4.48 & -0.64 & 244 & -0.095 & 94 \\
\hline Cotton & 1.91 & 1.95 & -0.15 & 48 & -0.078 & 96 \\
\hline Legumes & 0.61 & 0.68 & 0.01 & 29 & 0.016 & 96 \\
\hline Other crops & 0.11 & 0.19 & 0.04 & 14 & 0.364 & 96 \\
\hline Total area & 9.38 & 6.03 & -0.74 & 268 & -7.89 & 94 \\
\hline
\end{tabular}


The different components were calculated for several surface area variables, leading to the RTE of the different methods. Table 4 presents their values calculated for different area variables such as plot size and average crop area per farm. The first three columns present the mean of the different surface area variables $(\mu)$, the mean error or bias ( $B$, as calculated in the previous section), and BR. The fourth and fifth columns present the relative standard deviation of the area variable $\left(\mathrm{SR}_{x}\right)$ and of the observational error $\left(\mathrm{SR}_{e}\right)$, while the next column presents the relative covariance between area and error $\left(\mathrm{SR}_{x e}\right)$. Assuming area measurement has no observational errors, the total error for measuring equals the sampling error $\mathrm{SR}_{x} / \sqrt{n}$ or $\mathrm{RTE}_{x}$.

The total survey error for estimating surface area, on the other hand, needs to include variance and covariance of the observational error, according to Eq. (9), which results in $\mathrm{RTE}_{y}$, presented in the last column of Table 4.

The results for the plot size are presented in the first row of Table 4. The standard deviation is higher than the mean $\left(\mathrm{SR}_{x}=130 \%\right)$, but the sample size at the plot level is very large $(n=1080)$, resulting in a small sampling error $\left(\mathrm{RTE}_{x}\right)$ of just $4 \%$ of the mean plot size. The mean observational error is also substantial $(\mathrm{BR}=11 \%)$ and highly variable $\left(\mathrm{SR}_{e}=61 \%\right)$, but the covariance is negative. All these elements, combined in Eq. (9), result in a relative total error, $\left(\mathrm{RTE}_{y}\right)$ of $12 \%$.

For the total area per farm, the bias (BR) as well as the standard deviation $\left(\mathrm{SR}_{x}\right)$ are much smaller, but so is the sample size. The total survey error from measuring $\left(\mathrm{RTE}_{x}\right)$, equal to the sampling error, is $6.6 \%$. The bias is relatively small $(8 \%)$, as is the standard deviation (64\%), and the covariance between area and observational error is again negative. As a result, the total error of the estimation, $\mathrm{RTE}_{y}$, is $9.4 \%$ : not much higher than the total error of measuring $\mathrm{RTE}_{x}$, which is just the sampling error of $6.6 \%$. For the three principal groups of crops, similar results were obtained: the observation bias falls somewhere between $10 \%$ and $13 \%$ of the mean, which is slightly higher than the sampling error of $7-11 \%$. The measurement of plots results in a gain of accuracy of $2-4 \%$ as compared with farmers' estimate after visual inspection.

\subsection{Comparing cost and accuracy of the two measuring methods}

During the survey, enumerators visited on average four farms per day to estimate all their fields in collaboration with the head of the household. The physical measurement of those same fields, on the other hand, required a team of two enumerators, who would on an average day measure the fields of one and a half farms. The salaries and per diems of the enumerators were about US $\$ 7$ per person per day, while the transportation cost were about US $\$ 4$ per motorcycle per day. The team of measurers used one motorcycle for two enumerators, while the FSR village workers, who did the estimation, all used an individual motorcycle. The difference in grade and compensation package between temporary and long-contract workers can be ignored here, and overhead cost, data entry and analysis costs can be considered equal for both methods.

Table 5 shows the difference in variable costs of the two methods. For 1068 plots on 96 farms in 12 villages, physical measurements cost \$2328 and result in a precision 
Table 4

Components of relative total error, calculated for measuring $\left(\mathrm{RTE}_{x}\right)$ and estimating $\left(\mathrm{RTE}_{y}\right)$ for several key area variables

\begin{tabular}{|c|c|c|c|c|c|c|c|c|c|}
\hline \multirow[t]{2}{*}{ Variable } & \multirow{2}{*}{$\begin{array}{l}\text { Mean } \\
\mu \text { (ha) }\end{array}$} & \multirow{2}{*}{$\begin{array}{l}\text { Bias } \\
B \text { (ha) }\end{array}$} & \multirow{2}{*}{$\begin{array}{l}\text { Relative } \\
\text { bias BR }(B / \mu)\end{array}$} & \multirow{2}{*}{$\begin{array}{l}\text { Relative } \\
\operatorname{SD~SR}_{x}(\sigma / \mu)\end{array}$} & \multirow{2}{*}{$\begin{array}{l}\text { Relative SD of } \\
\text { observational } \\
\text { error } \mathrm{SR}_{e}\end{array}$} & \multirow{2}{*}{$\begin{array}{l}\text { Relative } \\
\text { covariance } \mathrm{SR}_{x e}\end{array}$} & \multirow[t]{2}{*}{$n$} & \multicolumn{2}{|l|}{ Relative total error } \\
\hline & & & & & & & & Measuring $\mathrm{RTE}_{x}$ & Estimating $\mathrm{RTE}_{y}$ \\
\hline Plot size & 0.82 & -0.093 & -0.114 & 1.30 & 0.61 & -0.45 & 1080 & 0.040 & 0.119 \\
\hline Total area/farm & 9.38 & -0.074 & -0.079 & 0.64 & 0.29 & -0.12 & 94 & 0.066 & 0.094 \\
\hline Area in cotton & 1.91 & -0.15 & -0.078 & 1.02 & 0.25 & -0.11 & 96 & 0.104 & 0.124 \\
\hline Area in cereals & 6.70 & -0.64 & -0.095 & 0.67 & 0.36 & -0.18 & 94 & 0.069 & 0.107 \\
\hline Area in legumes & 0.61 & 0.01 & 0.024 & 1.11 & 0.47 & -0.14 & 96 & 0.114 & 0.114 \\
\hline Area in other crops & 0.11 & 0.04 & 0.374 & 1.76 & 1.29 & -0.14 & 96 & 0.180 & 0.432 \\
\hline
\end{tabular}


Table 5

The cost of measuring and estimating the fields of 96 farms in 12 villages in Mali

\begin{tabular}{lcc}
\hline & Measuring & Estimating \\
\hline Number of farms & 96 & 96 \\
Number of plots & 1068 & 1068 \\
Man days needed for the survey & 248 & 32 \\
Salary cost (\$/man day) & 7.22 & 7.22 \\
Transport cost (\$man day) & 2.17 & 4.35 \\
Total variable costs (\$) & 2328 & 370 \\
Total variable cost/farm (\$) & 24.25 & 3.85 \\
Total error for farm size (RTE as \% of mean) & 6.6 & 9.4 \\
Total error for plot size (RTE as \% of mean) & 4.0 & 11.9 \\
\hline
\end{tabular}

of $6.6 \%$. Estimating the same areas after visual inspection only costs $\$ 370$, but the resulting error is $9.4 \%$. Does the extra accuracy of $2.6 \%$ justify the extra cost of $\$ 1958$ ? Decision theory suggests that the information cost be compared with the possible losses caused by taking the wrong decision based on faulty information. In practice, however, it is more relevant to compare the extra $2.4 \%$ accuracy to the use of the survey results with alternative allocations of the money for the research institute, such as hiring an analyst or buying a computer.

\subsection{Minimizing error under research budget constraints}

As an alternative, the money saved by the cheaper estimation method can be used to increase the sample size. Therefore, we should compare the two methods, not at equal sample sizes, but at equal budgets. For ease of reasoning and calculation, assume simple random sampling and only consider variable costs. The maximum sample size for a given budget is then calculated by dividing the budget by the cost per farm, \$24 for measuring and \$4 for estimating after visual observation. Using the data from Table 5 in Eq. (9), the RTE can now be calculated for both methods at the given budget. By repeating the calculations at different budget levels, the trade-off between costs and accuracy can be quantified, as demonstrated in Fig. 3 for the estimation of total farm area. It shows how, at low budgets, the estimation method is actually more accurate than physical measurement because of the very low cost per farm (\$4). A total error of less then $10 \%$ can be obtained with a sample of 67 farms at a cost of $\$ 260$. Increasing the sample size reduces the sampling error, but not the bias, which is quite large $(7.9 \%)$. Therefore, once the sample size has reached 200 , obtaining a total error of $8.6 \%$ with budget of $\$ 800$, increasing its size can hardly reduce the total error any further.

Since measuring is more expensive, small budgets only permit small samples with high sampling errors. Bias, however, is independent of sampling size. Therefore, cheaper methods with a higher bias can be more interesting for low budgets, since the sampling error of the expensive method will be higher than the bias of the cheap method. If the available budget increases, the more expensive method can be used on a larger scale, and the sampling error becomes less than the bias of the cheaper 


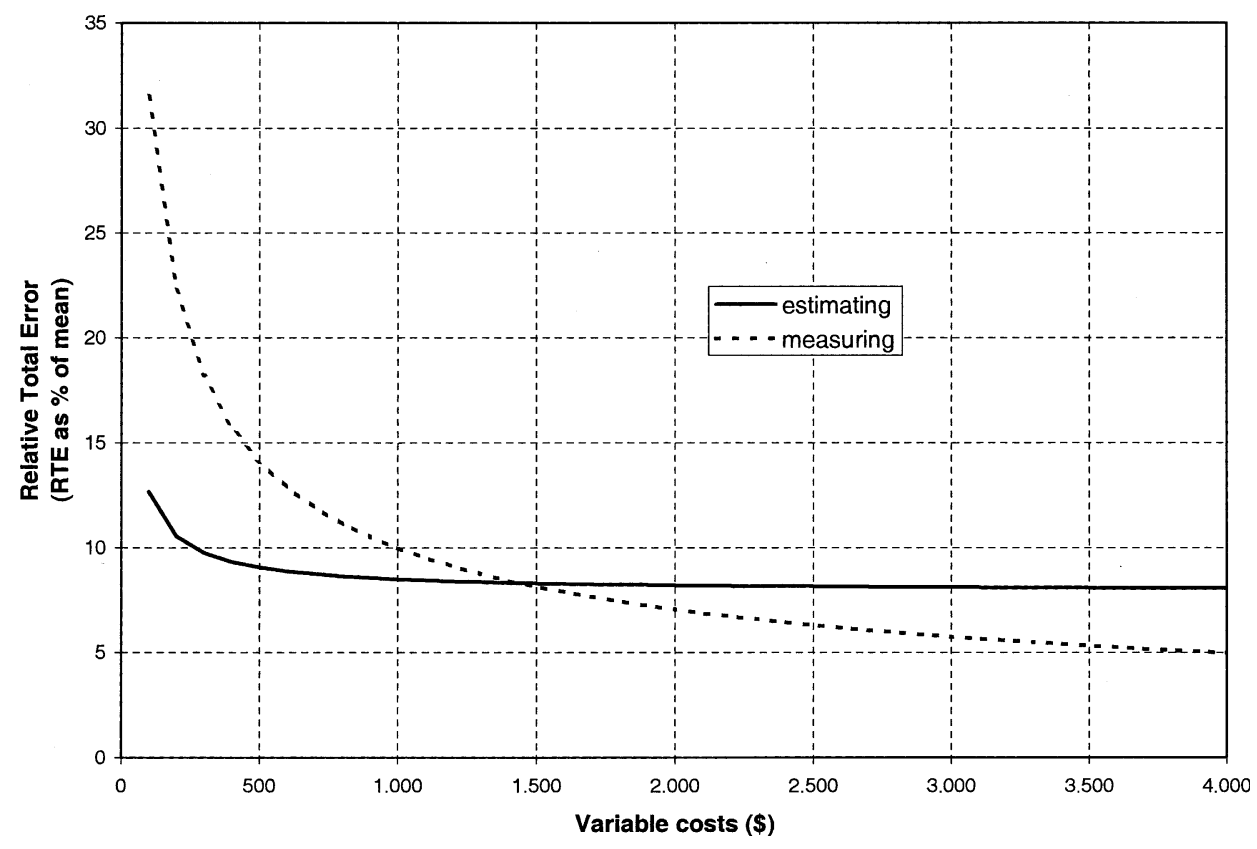

Fig. 3. The cost of accuracy in estimating total farm area.

method. In our example, starting at a budget of $\$ 1500$, the sampling error of measuring becomes less then the bias of estimating, so from here on, physical measurement is more cost efficient. Because the method is in principle unbiased, the total error keeps decreasing with increasing budget and sample size. However, since the total error is inversely proportional to the square root of sample size, this effect decreases gradually. At a budget of $\$ 4000$, a sample of 160 farms results in an RTE of $6 \%$. To reduce the error to 5\%, however, 100 more farms are needed at an extra cost of $\$ 2400$.

Although all variables follow curves similar to Fig. 3, their particular shape depends on their variance and bias parameters. Cotton area per farm, for example, has a substantially higher variance than total area, so to obtain a $10 \%$ error the cotton areas of 180 farms needs to be estimated, at a cost of $\$ 700$. Similarly, the budget point at which the lines cross and where physical measurement becomes more cost efficient than the estimation method, depends on those variance and bias elements that constitute total error, as presented in Table 5. For cotton area per farm, for example, physical measurement only becomes more cost efficient at a $\$ 3500$ budget, and to reach 6\% accuracy, 1800 farms are needed, at a cost of $\$ 7000$.

\subsection{Towards a general formula for relative total error}

The RTE calculated here depend on the parameters used, which are specific to the survey. Unfortunately, few methodological studies are available to provide the parameters needed to use the methodology presented. Still, simpler and more approx- 
Table 6

Decomposition of the variance of the estimated values

\begin{tabular}{llllll}
\hline Variable & Mean $\mu$ (ha) & \multicolumn{2}{l}{ Relative standard error } & \multicolumn{2}{l}{\begin{tabular}{l} 
Relative covariance \\
\cline { 2 - 5 } $\mathrm{SR}_{x e}$
\end{tabular}} \\
\cline { 2 - 5 } & & Estimating $\mathrm{SR}_{y}$ & Measuring $\mathrm{SR}_{x}$ & Error $\mathrm{SR}_{e}$ & \\
\hline Plot size & 0.82 & 1.08 & 1.30 & 0.61 & -0.45 \\
Total area/farm & 9.38 & 0.50 & 0.64 & 0.29 & -0.12 \\
Area in cotton & 1.91 & 0.94 & 1.02 & 0.25 & -0.11 \\
Area in cereals & 6.70 & 0.47 & 0.67 & 0.36 & -0.18 \\
Area in legumes & 0.61 & 1.08 & 1.11 & 0.47 & -0.14 \\
Area in other crops & 0.11 & 2.12 & 1.76 & 1.29 & -0.14 \\
\hline
\end{tabular}

imate methods can easily be derived. From the assumptions of the linear error model (Eqs. (2) and (5)) it follows that:

$$
V(\bar{y})=V(x+e)=\sigma_{x}^{2}+\sigma_{x}^{2}+\sigma_{x e} .
$$

By dividing all terms by the mean of the variables, we obtain

$$
\mathrm{SR}_{y}=\sqrt{\mathrm{SR}_{x}^{2}+\mathrm{SR}_{e}^{2}+2 \mathrm{SR}_{x e}} .
$$

The values of those four parameters are represented in Table 6 for all the variables previously treated. It shows that for the major variables (plot size, farm size, and area under the major crops), the relative standard deviation of the estimated values $\left(\mathrm{SR}_{y}\right)$ is smaller than the relative standard deviation of the real values $\mathrm{SR}_{x}$, usually called coefficient of variation $(\mathrm{CV})$. The reason is that the variance of the error $\left(\mathrm{SR}_{e}\right)$ is usually small, and the correlation of the error with the real value $\left(\mathrm{SR}_{x e}\right)$ is large and negative. We can now use this effect to simplify the total error formula:

$$
\begin{aligned}
\operatorname{RTE}(\bar{y}) & =\frac{1}{\bar{X}} \sqrt{V(\bar{y})+B^{2}}=\sqrt{\frac{\sigma_{y}^{2}}{n \bar{X}^{2}}+\frac{B^{2}}{\bar{X}^{2}}} \\
& =\sqrt{\frac{\mathrm{SR}_{y}^{2}}{n}+\mathrm{BR}^{2}} \leqslant \sqrt{\frac{\mathrm{SR}_{x}^{2}}{n}+\mathrm{BR}^{2}} .
\end{aligned}
$$

Therefore, the CV of the surface area variable and the relative bias of the method used are sufficient for a conservative estimate of accuracy with the RTE

$$
\operatorname{RTE}(\bar{y}) \leqslant \sqrt{\frac{\mathrm{SR}_{x}^{2}}{n}+\mathrm{BR}^{2}}=\sqrt{\frac{\mathrm{CV}^{2}}{n}+\mathrm{BR}^{2}} .
$$

The values for this last formula are easily calculated and tabulated in a spreadsheet, to visualize the effect of the relative variance, the sample size and the relative bias on relative total error. The calculations presented in Table 4 indicate that CVs (or $\mathrm{SR}_{x}$ ) for total farm area and the area in the most important crops fall somewhere between $67 \%$ and $111 \%$. For the same variables, the bias for area estimation falls between $2.4 \%$ and $9.5 \%$. 


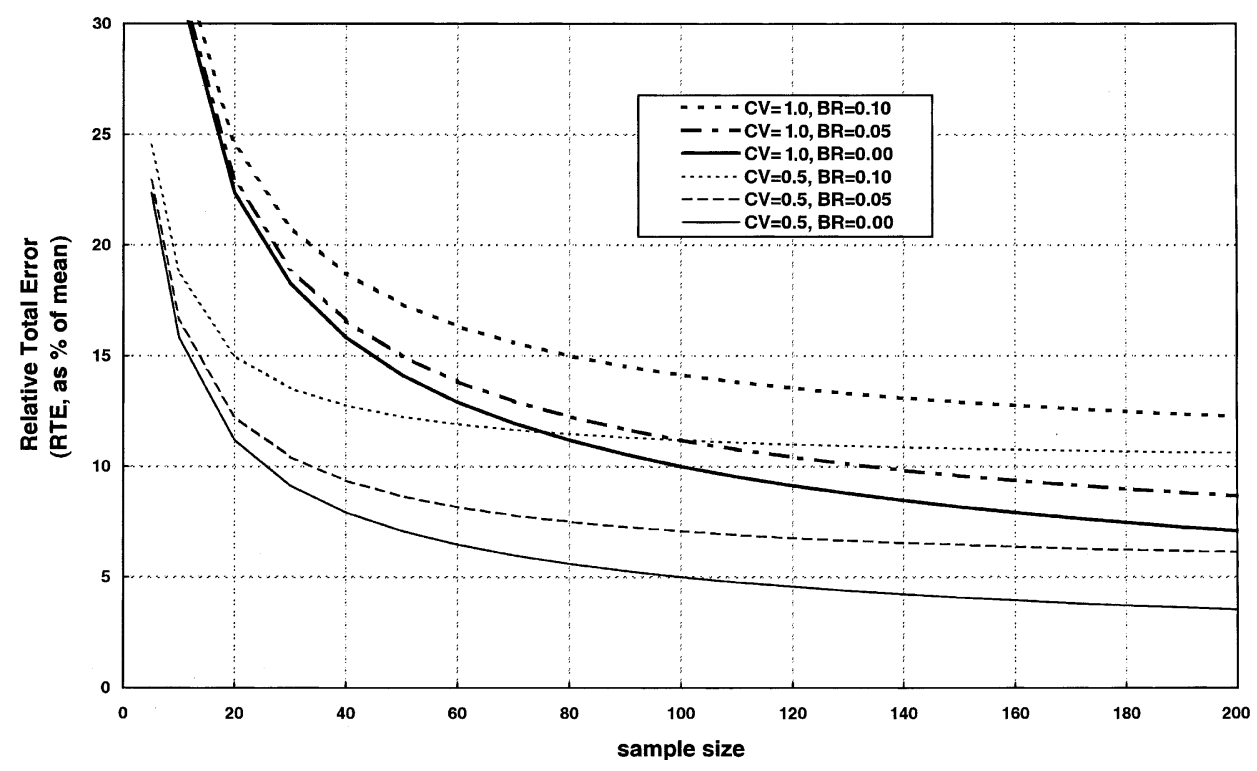

Fig. 4. Accuracy of a biased estimator.

Fig. 4 shows the RTE for the benchmark CVs of $50 \%$ and $100 \%$ and for relative biases of $0 \%, 5 \%$ and $10 \%$. It shows that a variable with a CV of $50 \%$, measured without bias, can be estimated with an accuracy of 5\% from a sample size of $n=100$. The curves show how increasing the sample size makes the relative total error converge towards the relative bias. It also demonstrates that the total error for biased estimators, even for variables with small CVs, hardly decreases any more above sample sizes of 100 .

\section{Conclusion}

The results of the present study show the potential of a new surface area estimation method, which combines surface area estimation by the farmer with visual inspection by the enumerator. The accuracy of this method is reasonably good, or at least much better than the farmers' estimates reported by FAO (1982), and considerably cheaper than measuring the same plots using the topofil method. The method's observational error is relatively small, with an average relative bias of $-11 \%$ for the estimation of plot size. The observational errors have a distinct negative covariance with the plot size, so when plot sizes are aggregated into surface area per farm, either by crop or total farm area, average errors are very reasonable, with an average or bias of between $2 \%$ and $-9 \%$ for the most important variables. However, if plot size information is important, the large observational error of farmers' estimate might be of concern, especially for the smaller plots. 
The accuracy of the method, as measured by RTE, is $9.4 \%$ for estimating average farm size with a sample of 96 farms, higher than the RTE of $6.6 \%$ for physical measurement. However, the estimation method saves more than US\$2000, making it well suited for low-budget surveys or surveys where accuracy is not that important.

Taking advantage of the fact that the variance of estimated values is lower than the variance of actual values, a simple general formula can be derived, using only an estimate of the coefficient of variation $\mathrm{CV}$ and of the relative bias BR. The formula, with parameters calculated for a number of surface area variables, can be used to predict the accuracy of different options for future surveys. This, we hope, will help to make rational decisions based on comparing accuracy with cost.

The generalization of Eq. (11) only holds for similar variables with low variance of the observational error, and negative correlation between error and true values. Secondary data can provide approximations of CVs of those variables, although unfortunately these variances or CVs are unfortunately rarely published in reports on agricultural statistics. The bias of measurement methods is also rarely estimated, but some reports exist. An overview of methods for measuring crop production is given by Poate (1988), while Murphy et al. (1991) summarize the available studies on farmers' estimates of crop production. The results of the present study, however, show that the whole variance-covariance matrix of errors and true values needs to be estimated to make useful comparisons through an accuracy measure such as the RTE.

Once estimates for this variance-covariance matrix become available, the same methodology can be applied for similar analysis to more complex sampling schemes or estimators. More general formulas for RTE can be developed based on formula (4), which are applicable to most sampling schemes and all linear estimators (De Groote, 1996).

Finally, this study confirmed that farmers, within a proper framework and following a well-defined methodology, can provide reasonably accurate estimates at very low costs. The analysis shows, however, that these surveys need to be accompanied by methodological studies to assess the accuracy of new methods used. In particular, the methodology should now be used to estimate the accuracy of global positioning systems (GPSs) for area estimation. Unfortunately, at the time of this study, these instruments were not yet available in Mali, but the example presented here clearly shows the convenience of the RTE to assess their accuracy.

\section{Acknowledgement}

The authors thank Demba Kebe, the Farming Systems Research team leader of Sikasso; Toon Defoer, head of the technical assistance team, Mme Sanata Sanogo Kone, research assistant; the coordinators of the regional FSR offices and the enumerators for their help and support. We also thank Bob Carsky, Steve Franzel, George Owuor and two reviewers for their remarks and suggestions. This study was executed within the FSR project of Sikasso, and financed by the Dutch Agency of Development Cooperation (DGIS). 


\section{References}

Ajayi, O.C., Waibel, H., 2000. How accurate are farm size estimates obtained from smallholder farmers in West Africa? Lessons from Côte d'Ivoire. In: Renard, G., Krieg, S., Lawrence, P., von Oppen, M. (Eds.), Farmers and Scientists in a Changing Environment: Assessing Research in West Africa. Margraf Verlag Publishers, Weikersheim, Germany, pp. 543-550.

Casley, D.J., Lury, D.D., 1981. Data Collection in Developing Countries. Oxford University Press, Cambridge, MA.

Cochran, W.G., 1977. Sampling Techniques. Wiley, New York 428 p.

DNSI, 1996. Enquête Agricole de Conjoncture, Campagne 1995/1996, Résultats Définitifs. Direction Nationale de la Statistique et de l'Informatique, Bamako.

De Groote, H., 1992. Optimal survey design in evaluating rural development projects. Unpublished Ph.D. Dissertation, University of Wisconsin, Madison, WI.

De Groote, H., 1996. Optimal survey design for rural data collection in developing countries. Quart. J. Int. Agric. 35 (2), 163-175.

FAO, 1982. Estimation des superficies cultivées et des rendements dans les statistiques agricoles. Etude FAO: développement économiqeu et social 22. Food and Agriculture Organization, Rome.

Giraudy, F., 1994. Résultats de le l'Enquête Agricole Permanente 93/94, Annuaire Statistique. Compagnie Malienne pour le Développement des Textiles, Bamako.

Groves, R., 1989. Survey Errors and Survey Costs. Wiley, New York.

Idaikkadar, N., 1979. Agricultural Statistics: A Handbook for Developing Countries. Pergamon Press, Oxford.

Kébé, D., Djouara, H., Traoré, O., De Groote, H., 1997. Suivi-évaluation permanent: Expérience de l'ESPGRN-Sikasso. Note Méthodologique, Institut d'Economie Rural, Sikasso, Mali.

Kelly, V., Hopkins, J., Reardon, T., Crawford, E., 1995. Using micro data to improve the measurement and analysis of african agricultural productivity. Department of Agricultural Economics Staff Paper No. 95-24, Michigan State University, East Lansing, MI.

Ministère de la Coopération, 1980. Mémento de l'Agronome. Ministère de la Coopération, République Française, Paris, 1598p.

Murphy, J., Casley, D., Curry, J., 1991. Farmers' Estimations as a Source of Productiom Data: Methodological Guidelines for Cereals in Africa. World Bank, Washington, DC.

Poate, D., 1988. A review of methods for measuring crop production from smallholder producers. Exp. Agric. 24 (1), 1-14.

Poate, C.D., Casley, D.J., 1985. Estimating Crop Production in Development Projects, Methods and their Limitations. World Bank, Washington.

Smith, J., Walker, P., Oyewole, T. 1988. Accuracy of farmer estimate of field size, RCMD Annual Report. IITA, Ibadan.

Verma, V., Marchant, T., Scott, C., 1988. Evaluation of Crop-cut Methods and Farmer Reports for Estimating Crop Production, Results of a Methodological Study on Five African Countries. Longacre Agricultural Development Centre Limited, London. 